\title{
BMJ Open Maternal experience of intermittent kangaroo mother care for late preterm infants: a mixed-methods study in four postnatal wards in China
}

\author{
Bo Zhang, ${ }^{1}$ Jieya Yue, ${ }^{1}$ Zhiying Duan, ${ }^{1}$ Yingxi Zhao (D) , ${ }^{2}$ Sarah Williams, ${ }^{3}$ \\ Limin Huang, ${ }^{4}$ Xiaoqin Zhang, ${ }^{5}$ Wenli Wu, ${ }^{6}$ Lin Zhang, ${ }^{7}$ Jun Liu, ${ }^{1}$ Gengli Zhao (D) ${ }^{1}$
}

To cite: Zhang B, Yue J, Duan Z, et al. Maternal experience of intermittent kangaroo mother care for late preterm infants: a mixed-methods study in four postnatal wards in China. BMJ Open 2021;11:e050221. doi:10.1136/ bmjopen-2021-050221

- Prepublication history and additional supplemental material for this paper are available online. To view these files, please visit the journal online (http://dx.doi.org/10.1136/ bmjopen-2021-050221)

Received 18 February 2021 Accepted 13 August 2021

Check for updates

(C) Author(s) (or their employer(s)) 2021. Re-use permitted under CC BY-NC. No commercial re-use. See rights and permissions. Published by BMJ.

${ }^{1}$ Peking University First Hospital, Beijing, China

${ }^{2}$ Nuffield Department of Medicine, University of Oxford, Oxford, UK

${ }^{3}$ Save the Children UK, London, UK

${ }^{4}$ Hunan Provincial Maternal and Child Health Hospital, Hunan,

China

${ }^{5}$ Northwest Women \& Children Hospital Department of Obstetrics, Shaanxi, China

${ }^{6}$ Linyi Maternity and Child Health Hospital, Shandong, China

${ }^{7}$ Save the Children, Chengdu, China

Correspondence to

Dr Gengli Zhao;

zhaogengli@sina.com

\section{ABSTRACT}

Objective To describe how mothers of late preterm infants experienced the provision of intermittent kangaroo mother care (KMC) in four postnatal wards in different hospitals in China, under a pilot KMC project.

Design A concurrent mixed-methods approach incorporating quantitative maternal questionnaires and qualitative semistructured interviews.

Setting Four postnatal wards in level-III hospitals based in different provinces of Southeast and Northwest China. Participants All 752 mothers who provided intermittent $\mathrm{KMC}$ to their late preterm newborns in the four participating postnatal wards consented to participate in the study (quantitative component), as well as six nurses, two obstetricians and two mothers from two of the participating postnatal wards (qualitative component). Outcome measures Maternal KMC experiences during a hospital stay, patients' perceptions of KMC initiation, processes, benefits and challenges.

Results Most mothers had not heard of KMC before being introduced to it in the postnatal ward. On average, mothers and newborns stayed in postnatal wards for 3.6 days; during their stay, mothers provided an average of $3.5 \mathrm{KMC}$ sessions, which is an average of 1.1 sessions a day. Each KMC session lasted an average of $68 \mathrm{~min}$, though there was much variation in the length of a session. Common reasons given for discontinuing a KMC session included restroom use, infant crying and perceived time limitations. Some mothers would have preferred to provide KMC for longer periods of time and nurses encouraged this. Most mothers experienced no difficulty providing KMC, received support from family and medical staff and intended to continue with KMC postdischarge.

Conclusion In order to improve the maternal experience of $\mathrm{KMC}$, it is recommended that raising awareness of KMC should be included in antenatal care and after birth. Longer periods of KMC provision should be encouraged, greater privacy should be provided for mothers providing KMC in postnatal wards and family members should be encouraged to support KMC.

\section{BACKGROUND}

Kangaroo mother care (KMC) originated in Bogota, Colombia in the 1970s. It has been shown to reduce neonatal mortality and
Strengths and limitations of this study

- Our study is the first to document the maternal experience and practice of kangaroo mother care (KMC) in China's postnatal wards using a mixedmethod approach.

- Our study has implications for clinical practices in China, including informing the development of national guidelines and aiding KMC scale-up.

- All hospitals included in the study are tertiary hospitals located in major urban cities, and KMC experiences in lower-level healthcare facilities could be different.

- The sample size for our qualitative research was relatively small, and a larger sample size would have given us a more reliable representation of maternal experience.

morbidity and increase breastfeeding rates when compared with conventional care. ${ }^{1}$ It is recommended by $\mathrm{WHO}$ as the standard of care for stable preterm newborns, and is defined as early, continuous and prolonged skin-to-skin contact between the preterm or low birthweight infant and caregiver, exclusive breast feeding and early hospital discharge accompanied by adequate follow-up and support. ${ }^{2}$

In 2014, there were an estimated 1168 126 preterm births in China, representing $7.8 \%$ of global preterm births. China is the second highest contributor to global preterm birth numbers, ${ }^{3}$ but, despite this, $\mathrm{KMC}$ is not widely practised or promoted in China. A few hospitals piloted KMC to varying degrees, but there has been no evidence of its routine practice $^{4}$ and no standardised guideline had been developed. Major barriers include highly restrictive access or no access for parents to their newborns who are cared for in neonatal intensive care units (NICUs), as well as limited hospital stays (1-4 days) for 
mothers and late preterm newborns cared for in postnatal wards. ${ }^{45}$ Since 2014, the Premature Birth Intervention Programme has raised awareness and promoted the uptake of KMC among its network of 50 hospitals, and has initiated a structured pilot of KMC in eight self-selected hospitals. The pilot implemented research focused on assessing the feasibility of adopting intermittent KMC in NICUs and postnatal wards, with the hope that evidence and learning could be used to inform the development of a national guideline and promote the scale-up of KMC in hospitals outside of the programme. Intermittent KMC refers to recurrent but not continuous skin-to-skin contact between a mother and infant, alternated with conventional care. ${ }^{2}$

In this paper, we aim to describe how mothers of late preterm infants experienced provision of KMCin postnatal wards under this pilot project. By better understanding how mothers perceived KMC initiation, processes, benefits and challenges, we can revise and adapt our procedures and ensure that the national guideline considers different local traditions, values and contexts.

\section{METHODS}

This study was part of a larger study with implementation research focused on KMC in China, conducted from March 2018 to March 2019. In this study, we used a pragmatic concurrent mixed-methods approach including quantitative maternal questionnaires and qualitative semistructured interviews. Detailed information on our approach to quantitative and qualitative data collection has been described in other publications. ${ }^{56}$ The focus of the current analysis differs from our previously published papers, which focused on breast feeding, barriers and facilitators for $\mathrm{KMC}$ adoption.

\section{Study setting}

The research was conducted in four level-III hospitals based in different provinces of Southeast and Northwest China. The average number of births per month in the hospitals ranged from 500 to 1000 . In these hospitals, when a woman gives birth to a late preterm newborn, born between 34 and 36 weeks and 6 days of pregnancy, the current practice is for a paediatrician to assess the newborn, if stable, the newborn will be admitted to the postnatal ward with their mother. As part of the pilot technical training, sessions on KMC and data collection were provided to medical and nursing staff working in wards that were participating in the study. Intermittent KMC was recommended to all mothers and families of late preterm infants that were admitted to participating postnatal wards.

\section{Data collection}

During the study period, 1007 mothers were admitted to postnatal wards with late preterm newborns, and of these, 752 agreed to provide KMC during their hospital stay and also agreed to take part in the study. Data were collected by designated nurses who received training in data collection, entry and verification, as part of their routine work. All designated nurses signed the confidentiality agreement. Data collected included maternal sociodemographic information (age, educational attainment, occupation), basic obstetric and neonatal information (obstetric: parity, presence of obstetric complications, mode of delivery; neonatal: birth weight and gestation at birth). Nurses also recorded the length of hospital stays and details concerning each KMC session that as provided (start and end time, duration and reason for stopping) (the data collection form is available in online supplemental material 1).

Before hospital discharge, a quantitative survey exploring the maternal attitudes and experiences with KMC was conducted (the survey questionnaire is available in online supplemental material 1). This included whether mothers had encountered any difficulties providing $\mathrm{KMC}$, their preferred duration of and time of day for providing KMC, whether they received family, medical and nursing staff support, and whether they intended to continue providing KMC after discharge.

After discharge, nurses phoned mothers on the 42nd day postbirth and administered a short survey asking whether they had continued to provide intermittent $\mathrm{KMC}$, they enquired about the frequency with which the mothers were providing KMC.

Qualitative data collection was conducted by six research assistants who volunteered for the role and were also hospital staff and project officers experienced with KMC. All research assistants received training on qualitative research, observation and interview techniques prior to data collection, and were involved in the collection, transcription and analysis of data.

Qualitative data were collected using semistructured individual interviews conducted in two of the study postnatal wards from August to September 2018. The two postnatal wards were selected based on convenience, as they were postnatal wards providing KMC in the same hospital as neonatal units involved in the larger study. In each ward, the head nurse was assigned as the study coordinator for that site and assisted in the recruitment of a mix of obstetricians, nurses and parents who had experience with intermittent $\mathrm{KMC}$ for interview purposes. Individual interviews were conducted over 1-2 days in private rooms within each ward. Each interview lasted about 30-40 min, and all the interviews were conducted in Mandarin and recorded with participants' consent. Participants were not compensated for the interviews. Interviews with obstetricians and nurses gathered their perspectives on $\mathrm{KMC}$ in postnatal wards, and the interview questions were informed by the Consolidated Framework for Implementation Research. The interviews focused on mothers' knowledge of $\mathrm{KMC}$, their confidence in practising $\mathrm{KMC}$, and the perceived benefits and barriers of KMC practice. Following pilot interviews and discussions within the research team, the interview questions were revised to combine broad, open-ended questions with targeted, 
prompting questions, to ensure that they could be easily understood by study participants.

\section{Data analysis}

For quantitative data, we conducted descriptive analyses and presented the basic socio-demographic and delivery-related variables using unadjusted percentages. We presented maternal KMC experiences during hospital stays stratified by delivery mode (vaginal delivery or caesarean section), using unadjusted percentages for categorical variables and mean and SDs for continuous variables. We also compared the preferred and actual duration of KMC sessions from the perspective of the mothers. All statistical analyses were performed using Stata V.14, and test results were reported significant at a level of 0.05 .

Qualitative data from semistructured interviews were audiotaped and transcribed by the research assistants. For the current analysis, a general inductive analysis approach was used to focus on the experience and process of KMC in postnatal wards. ${ }^{7}$ Emerging themes and categories were developed by authors examining the transcript repeatedly. We presented the themes in chronological order starting with the introduction and initiation of $\mathrm{KMC}$, support during KMC, discontinuance of $\mathrm{KMC}$ and the perceived benefits and challenges of KMC. Data analysis was conducted in Mandarin and selected thematic codes were translated into English, which are presented in the results section.

\section{Patient and public involvement}

Patients and the public were not directly involved in the design, recruitment or conducting of this study. Our qualitative interview guide was amended after the pilot and iteratively adjusted based on participants' (including patients) feedback during initial interview rounds. Results from this study have been reported to participating hospitals in aggregate forms and written reports.

\section{RESULTS}

\section{Quantitative analysis}

All 752 women who provided KMC to their newborns agreed to take part in the study, and data from all these women were included in the analysis (table 1). Nearly half of the mothers had an educational attainment of university level and above, while $63.6 \%$ of the mothers were primiparous and $61.6 \%$ delivered through caesarean section. Most premature infants admitted to the postnatal ward had a gestational age between 36 weeks and 36 weeks and 6 days and a birth weight of $2500 \mathrm{~g}$ or above.

Table 2 shows mothers' experiences of KMC during their hospital stay, stratified by the mode of delivery. On average, mothers and newborns stayed on postnatal wards for 3.6 days and, during this time, mothers provided an average of 3.5 sessions of $\mathrm{KMC}$, an average of 1.1 sessions a day. The average length of each KMC session was 68 min, however, there was much variation between mothers.
Table 1 Basic sociodemographic of mothers who performed kangaroo mother care

\begin{tabular}{|c|c|c|}
\hline & $\begin{array}{l}\text { No } \\
(\mathrm{N}=752)\end{array}$ & $\%$ \\
\hline \multicolumn{3}{|l|}{ Age } \\
\hline$<30$ & 254 & 33.8 \\
\hline 30-34 & 294 & 39.1 \\
\hline$\geq 35$ & 204 & 27.1 \\
\hline \multicolumn{3}{|l|}{ Education attainment } \\
\hline High school & 222 & 29.5 \\
\hline College & 191 & 25.4 \\
\hline University and above & 339 & 45.1 \\
\hline \multicolumn{3}{|l|}{ Parity } \\
\hline Primipara & 478 & 63.6 \\
\hline Multipara & 274 & 36.4 \\
\hline \multicolumn{3}{|l|}{ Delivery mode } \\
\hline Vaginal delivery & 289 & 38.4 \\
\hline C-section & 463 & 61.6 \\
\hline \multicolumn{3}{|c|}{ Pregnancy-related complications } \\
\hline No & 327 & 43.5 \\
\hline Yes & 425 & 56.5 \\
\hline \multicolumn{3}{|l|}{ Birth weight } \\
\hline Normal ( $\geq 2500 \mathrm{~g})$ & 656 & 87.2 \\
\hline Low weight (<2500 g) & 96 & 12.8 \\
\hline \multicolumn{3}{|l|}{ Gestational age } \\
\hline 34-34+6 weeks & 2 & 0.3 \\
\hline $35-35+6$ weeks & 108 & 14.4 \\
\hline $36-36+6$ weeks & 642 & 85.4 \\
\hline
\end{tabular}

Values are $\mathrm{n}$ and \%.

Most mothers provided KMC for between 1 and 2 hours (45.6\%), or for less than 1 hour (39.9\%). Compared with those who gave birth vaginally, mothers who delivered through caesarean section had a longer average hospital stay (4.35 vs 2.42 days) and provided KMC less frequently on a daily basis ( 0.99 vs 1.26 sessions), but provided more KMC sessions during their hospital stay (3.98 vs 2.73 sessions). Common reasons mentioned for stopping a session of KMC included restroom use (45\% of mothers), infant crying $(36.2 \%)$ and a perceived prearranged time limitation (26.5\%). Most mothers received family support for KMC, as well as support from medical and nursing staff, which included adjusting the infants' position for KMC (mentioned by $90.1 \%$ of mothers who received KMC support), provision of guidance on how to observe the infant's condition $(77.5 \%)$, and feeding posture adjustments $(65.7 \%)$. Only $18.8 \%$ of mothers had heard of KMC before arriving to the postnatal ward. Those who had heard of KMC had heard about it from medical staff, media or the internet. Most mothers did not experience any difficulty performing KMC (89.0\%) 
Table 2 KMC experience during hospital stay

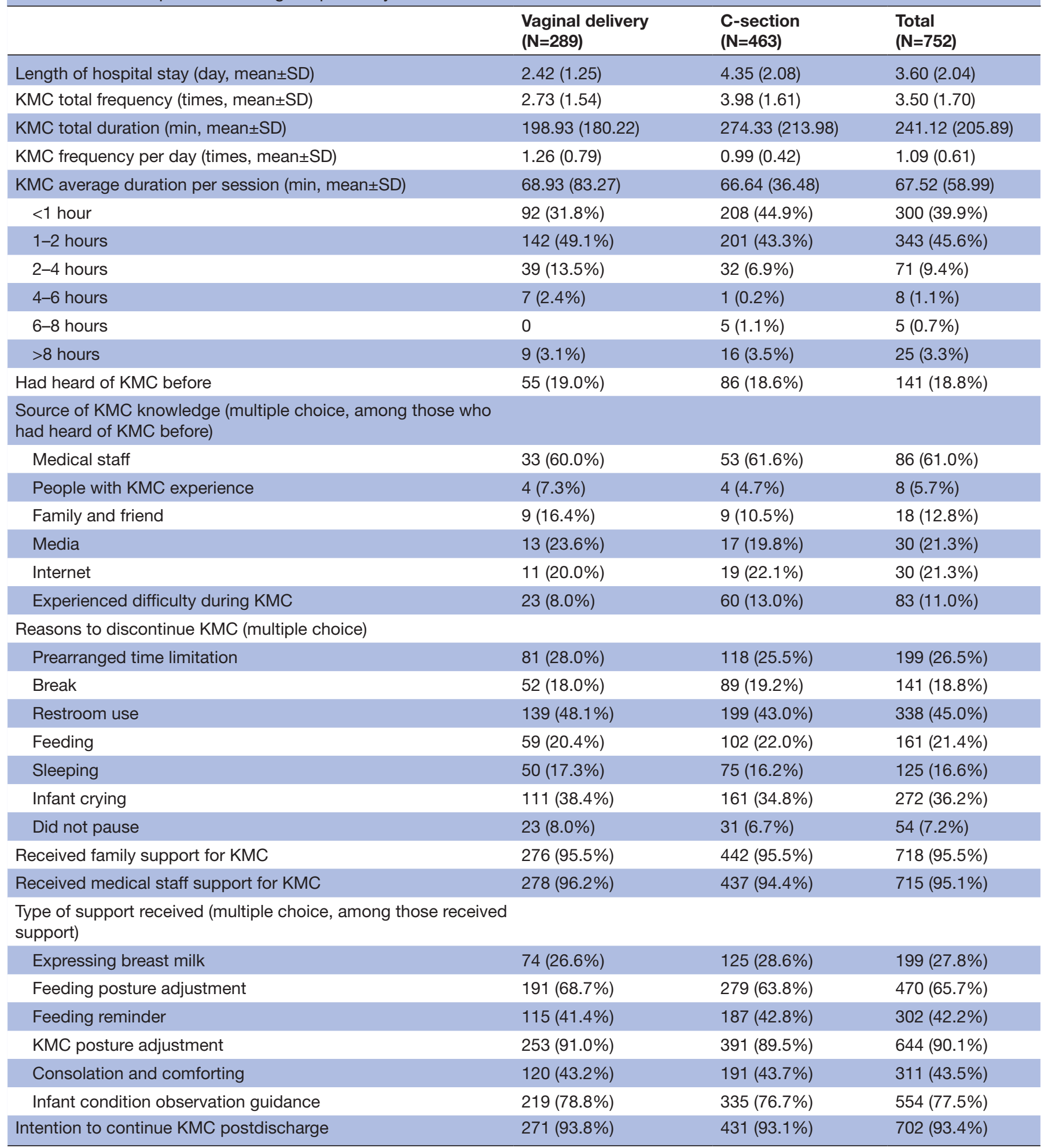

Values are $\mathrm{n}(\%)$.

$\mathrm{KMC}$, kangaroo mother care.

Table 3 shows mothers' preferences for the length of KMC sessions and the time of day they preferred to provide KMC. Over half of the mothers preferred to provide KMC for between 1 and 2 hours $(55.5 \%)$, while approximately a quarter $(26.7 \%)$ preferred a duration of less than 1 hour. Morning (34.4\%) and afternoon $(35.9 \%)$ were the times when most mothers preferred to provide KMC to their newborns.

At hospital discharge, the majority of mothers $(93.4 \%)$ intended to continue providing intermittent KMC. Of 
Table 3 Preferred KMC session duration and time of day

$\mathbf{N}=\mathbf{2 5 2}$

\begin{tabular}{ll}
\hline Preferred KMC duration & \\
$<1$ hour & $201(26.7)$ \\
$1-2$ hours & $417(55.5)$ \\
\hline $2-4$ hours & $77(10.2)$ \\
$4-6$ hours & $25(3.3)$ \\
$6-8$ hours & $8(1.1)$ \\
$>8$ hours & $2(0.3)$ \\
Others & $22(2.9)$ \\
\hline Preferred time of day & \\
\hline Morning & $259(34.3)$ \\
Noon & $93(12.4)$ \\
Afternoon & $270(35.9)$ \\
Evening & $106(14.1)$ \\
\hline Others & $24(3.2)$ \\
\hline
\end{tabular}

Values are $\mathrm{n}(\%)$.

$\mathrm{KMC}$, kangaroo mother care.

those mothers who were successfully followed up with $(\mathrm{n}=627)$ on the 42nd day after birth, 69.5\% $\quad(\mathrm{n}=436)$ reported that they had continued to provide intermittent KMC. The mean average frequency of KMC provision was 1.9 times a day, according to online supplemental material 2.

\section{Qualitative analysis}

A total of 10 semistructured interviews were conducted during August and September 2018 in two of the participating postnatal wards. Six out of the 10 interviewees were nurses involved in the provision of $\mathrm{KMC}$ on the ward, 2 were mothers providing intermittent $\mathrm{KMC}$ to their newborns and 2 were obstetricians -1 of whom had recently given birth to a preterm newborn and provided intermittent KMC. Four main themes emerged from the interviews, which are presented below.

\section{Introducing and initiating KMC}

The two hospitals operated differently in terms of when and how they introduce KMC to mothers. One hospital informed women about KMC after birth and on admission to the postnatal ward, and the other identified women at risk of preterm birth and informed them about KMC during antenatal care. Information focused on the benefits of KMC, including maintenance of body temperature, benefits for digestion and breast feeding and alleviation of postnatal depression. After informing mothers about KMC in the postnatal ward, consent for providing KMC was sought and some mothers preferred to discuss KMC with their families before deciding whether to provide it. Nurses emphasised that instead of directly introducing KMC and 'forcing' mothers to provide it, they would start by discussing the babies' prematurity, prompting mothers to ask about ways in which they could improve their babies' condition. Most mothers agreed to provide KMC, and a few refused due to pain associated with a caesarean delivery. After mothers agreed to perform KMC, nurses would prepare the mother and infant, which included reminding mothers to use the restroom and to clean their bodies in order to prepare them for KMC positions.

You just tell them what the benefits are, not too deep as they might not understand, just simple words. When they know that it's good for the infant and helps maintaining their vital signs, they are quite happy that it can help the infant and promote breast milk secretion, they are quite happy and like to try KMC. -01 (nurse)

If the infant turns out to be premature, I would definitely not say you need KMC. I would ask you how're you feeling and your condition as a mother...then, if your baby is a premature baby-if yes, what's your worry or concern? She might say that I am worried about feeding the baby and its growth. And then, I would say that besides regular neonatal care, there's something called KMC. It's a care strategy like a kangaroo.... they usually accept it well if I tell them step-by-step. If I just come and say that I am going to perform KMC on her infant, she might find it hard to accept. - 03 (nurse)

\section{Health workers support during KMC}

During each KMC session, nurses usually provided support for mothers and their infants, which included taking temperatures before each session, adjusting an infants' posture and observing them for any abnormalities. Interviewed nurses reported that when mothers were providing $\mathrm{KMC}$, they would spend time with them, support them and explain newborn care. This prompted more communication and interaction between nurses and mothers, contributing to better nurse-mother relationships.

Also, when the parents are performing KMC, we definitely will provide them with some new-born care guidance, and spend more time than average mothers. They will learn more professional knowledge. 05 (nurse)

Sometimes, sometimes I think that the posture is not very comfortable, and then sometimes I will ask the nurse to help me and adjust the posture, the baby will also feel more comfortable. - 06 (mother)

\section{Length of KMC and discontinuing KMC}

Mothers usually attempted to provide KMC for 1 hour per session, and some mothers provided KMC for 20-30 min, as they felt uncomfortable. Other common reasons mentioned for discontinuing a KMC session included mothers needing to use the restroom or having to eat, the baby crying, family visits or hospital procedures, such as hearing tests interrupting KMC provision. Nurses mentioned that mothers were instructed to try KMC for at least 1 hour each session, and would only attempt 
to provide 1 hour of $\mathrm{KMC}$ at a time. Nurses tried to encourage mothers to gradually increase the length of KMC provision, although it was challenging, as mothers and newborns did not stay in the postnatal ward for very long.

Sometimes KMC is discontinued, why? Because after the nurse set it up, the doctor may come and do some procedure on the baby, or people from the child development department may come and test the baby's hearing, then KMC is discontinued. Without those procedures, parents may persist longer. - 03 (nurse)

There's rarely any that could persist for three or four hours, mostly one to two hours. Mostly due to parental factors. Also likely because we first promoted and introduced KMC to be one hour session each, at least one hour, parents will then feel that one hour is enough...They feel like they have accomplished the task, the nurse told me one hour and I just need to do it for one hour. - 04 (nurse)

...They would ask when I should finish, we would say at least one hour, and then they would just aim for one hour. Also, people's perception has not changed, the grandparents heard the baby crying, and thought the baby must be feeling unformattable or painful. The grandparents would be nervous and say, let me hold the baby and discontinued the KMC.- 06 (nurse)

\section{Mother's perception of KMC benefits and challenges}

While there were many benefits nurses mentioned, there were not many observed benefits, primarily due to the limited length of hospital stays. Nurses claimed that babies receiving KMC cried less, grew faster and fed better, while also saying that parents seemed to be happier. When we asked nurses to recall what mothers said about the benefits of KMC, most mentioned better feeding and better emotional bonding.

...Frankly speaking, it's impossible to have an extremely significant difference...I think (the benefits) will show up when the infant grows up later, but it's hard to see a huge change in the short period.- 02 (obstetrician)

(The mother) said that when put in the trolley, the baby did not suck well and might sleep for three to five hours without waking up. As long as you put it on your skin for skin-to-skin contact, the baby shook its head and looked for food.- 04 (nurse)

I went through a C-section and when I tried KMC for the first time, I felt very warm as the baby stayed on me, I felt an emotional bonding and the baby felt safer. I also fed him my breast milk, stimulating the secretion is very important and the baby could eat well and grow faster.- 09 (mother)

There were several challenges mentioned, including lack of privacy during KMC and the continuation of KMC after hospital discharge.
It is best to have something covering (me), because there are too many people in the wards, there are also men, the children are naked, and my upper body is almost naked, which is not convenient. - 10 (mother)

(Parents) definitely think KMC is good, but the challenge is after discharge. When performing KMC during a hospital stay, there's health workers prompting, some people might not adhere to it after they go back home.- 08 (nurse)

\section{DISCUSSION}

Through analysing quantitative data collected from 752 mothers about their experiences with KMC, and qualitative data collected from 10 semistructured interviews with nurses, obstetricians and mothers, we investigated mothers' experiences of KMC during hospital stays in the postnatal wards. The majority of mothers found the experience of providing intermittent KMC to be positive and did not report any difficulties. Most reported that they intended to continue providing intermittent KMC postdischarge.

Our study focused on mothers of late preterm infants in the postnatal wards, the majority of whom had infants of normal birth weight ( $\geq 2500 \mathrm{~g})$ born at a $36-36+6$ weeks gestational age. Therefore, the length of their hospital stay was relatively short (3.60 days). Most low birthweight infants $(<2500 \mathrm{~g})$ were cared for in neonatal wards or in NICUs, according to hospital policy. Late preterm infants are at a higher risk of mortality and morbidity as opposed to term infants ${ }^{8}$ and have lower breastfeeding rates. ${ }^{9} \mathrm{KMC}$ could bring potential benefits to late preterm newborns and their mothers, including improved breastfeeding rates and better maternal recovery from birth-related fatigue. ${ }^{6}{ }^{10-14}$ The challenges of introducing, initiating and continuing KMC in postnatal wards could differ from those in neonatal units with early preterm newborns. Most of the mothers in our study had not heard of KMC before, and the length of their stay in postnatal wards was relatively short. Due to the larger size (over $2500 \mathrm{~g}$ ) of many of the newborns in our study, they may not have tolerated long periods of KMC as good as smaller newborns. ${ }^{15} 16$ Intermittent $\mathrm{KMC}$ was promoted instead of continuous $\mathrm{KMC}$, which has been more commonly used in resourcerich countries for varying periods of time. ${ }^{17} 18$

The majority of mothers provided intermittent KMC for less than 2 hours per session, once a day. While many mothers preferred to continue KMC for longer periods each time, a number of reasons led to these sessions being discontinued, including parents' discomfort, restroom use, infant crying, interruptions so that nursing care could take place and perceived time limitations. Another publication has described that providing $\mathrm{KMC}$ for longer sessions impedes activities including use of restrooms and eating, which are mentioned as common reasons for stopping KMC sessions. ${ }^{17}$ Although research suggests that KMC reduces crying in preterm infants, ${ }^{19}$ mothers 
and other family members (especially grandparents) in our study perceived crying as a sign of discomfort, and some discontinued KMC as a result. Incidents of infants crying could be due to their larger sizes, with most of the newborns in our study population weighing over $2500 \mathrm{~g}$. Compared with early preterm newborns, larger newborns are better able to regulate their own body temperature and may only tolerate short periods of KMC. ${ }^{15}$ Care procedures were another reason given for discontinuation of KMC, as doctors or medical staff needed to perform certain activities. With better planning and scheduling of medical procedures and checks, this discontinuation could be avoided. An important reason for discontinuing KMC sessions was the 1-hour 'time limitation,' commonly referred to by mothers and health workers as the standard duration. One hour of KMC was a minimum recommendation set by the Premature Birth Intervention Programme based on available literature and experiences from other countries. ${ }^{13}{ }^{17}$ Therefore, mothers may have felt obligated to persist with KMC for 1 hour, and many discontinued it after this length of time. Despite nurses encouraging mothers to increase the duration of KMC provision, this was challenging due to their extremely short hospital stays. Our survey of mothers' preferences for $\mathrm{KMC}$ practices indicated that mothers preferred KMC sessions to be between 1 and 2 hours in length, and preferred to provide KMC in the morning or afternoon. This information will inform our future work on intermittent KMC in postnatal wards in China.

Our findings indicate that most mothers did not experience difficulties performing $\mathrm{KMC}$, and, with support from nurses and family members, $\mathrm{KMC}$ was perceived to promote emotional bonding and improve feeding, as is commonly reported in literature. ${ }^{14}{ }^{17}$ Due to the provision of support for KMC, such as postural adjustment and guidance on newborn observation, nurses spent more time with mothers providing KMC and also provided information on other aspects of newborn care. Nurses felt that this increased maternal satisfaction and improved the relationship between nurses and mothers. However, it should be noted that providing KMC support increases the workload of health workers, especially nurses, ${ }^{5}$ which should be taken into account when planning for KMC implementation in postnatal wards.

While most mothers $(93.4 \%)$ intended to continue provision of KMC postdischarge, in the follow-up survey, taking place 42 days after birth, only $69.5 \%$ of mothers reported that they had continued to provide intermittent KMC. The difference between the number of mothers who intended to continue providing intermittent KMC after discharge and the number who required further investigation indicates a need for follow-up and support for KMC provision after hospital discharge.

Less than $20 \%$ of participating mothers had heard of KMC before being informed about it in the postnatal ward. Of those who had heard of KMC before, $61 \%$ reported medical staff as their source of information, and $20 \%$ had heard of KMC through the media and the internet. A lack of awareness of KMC among new mothers is not unique to China, as a study in India found that most mothers had not heard of KMC before being informed about it by a health worker. ${ }^{20}$ Some of the nurses interviewed in our study recommended introducing the concept of KMC during antenatal care, while others felt that this could be done immediately after delivery. KMC as a method of care for preterm newborns in China is still relatively new and its practice is not widespread. If more mothers and premature newborns are to benefit from $\mathrm{KMC}$, health workers have a key role to play in raising awareness, championing and informing parents.

Our study is the first to document maternal experiences and practice of KMC in China's postnatal wards and has implications for clinical practices in China, including informing the development of national guidelines and aiding KMC scale-up. We previously reported on barriers and facilitators to KMC adoption in five hospitals in China. ${ }^{5}$ That information is an aid to understanding the contextual and individual factors that influence the decision to initiate KMC. This paper is more relevant to the procedural aspects of KMC implementation and we hope it will inform practices that will improve mothers' experiences of KMC. For hospitals implementing or planning to implement intermittent KMC for late preterm newborns in postnatal wards, we suggest the following recommendations based on our findings.:

- Health workers should raise awareness of KMC among pregnant women and new mothers during antenatal care or after the birth of premature newborns.

- Media and internet platforms should be used to disseminate information and raise awareness about $\mathrm{KMC}$ and its benefits.

- Mothers should be encouraged to provide KMC for as long as possible and for as long as their newborns will tolerate it.

- KMC should be facilitated in postnatal wards by ensuring privacy for mothers and newborns during $\mathrm{KMC}$, adjusting the schedules of medical and nursing care procedures around $\mathrm{KMC}$, taking into account the effects of KMC on nurses' workload and, if possible, allocating additional staff to the ward and encouraging family members to support mothers to provide KMC.

Several limitations should be considered when interpreting these results. First, all hospitals included in the study are tertiary hospitals, including general teaching hospitals and maternal and child healthcare hospitals located in major urban cities. The experience of KMC in lower-level healthcare facilities may be different. Second, the sample size for our qualitative research was relatively small, and a larger sample size would have given us a more reliable representation of maternal experience. However, this was mitigated in some way by the triangulation of qualitative data with quantitative data.

Our study of late preterm infants cared for in the postnatal ward included newborns, most of whom did not have a low birth weight, because it was these premature 
newborns who were admitted to the postnatal wards. To reduce the separation of mothers and their stable late preterm newborns of low birth weight, there is a need for further research to assess the feasibility of caring for smaller preterm newborns in postnatal wards.

\section{CONCLUSION}

In this mixed-methods study, we found that intermittent KMC was provided to late preterm newborns in short sessions during a brief hospital stay in postnatal wards. A number of factors led to the discontinuation of KMC sessions, including interruptions for medical or nursing care tasks, restroom use, perceived set time limitations and infant crying. The majority of mothers did not report difficulties in providing KMC and intended to continue provision of intermittent KMC postdischarge. In order to improve the maternal experience of KMC, it is recommended that raising awareness of KMC should be included in antenatal care or soon after the birth of preterm newborns. Longer periods of KMC provision should be encouraged, more privacy should be provided in postnatal wards for mothers providing $\mathrm{KMC}$, and family members should be encouraged to support KMC.

Acknowledgements We thank Steve Wall for reviewing an earlier version of the manuscript.

Contributors BZ, JY and YZ conceived of the analysis. BZ, ZD, LH, XZ, WW and GZ led the data collection process. BZ and YZ produced the estimates, created figures and tables and wrote the first draft of the manuscript. ZD, SW, JY, JL and GZ provided critical feedback on the first draft of the manuscript. LZ managed the production process. All authors read and approved the final manuscript.

Funding This work was supported by Save the Children International (No. 34400085).

Competing interests None declared.

Patient consent for publication Not required.

Ethics approval Ethical approval was obtained by Peking University First Hospital Biomedical Research Ethics Committee (No. 2018(13)). All participants gave written consent or oral consent.

Provenance and peer review Not commissioned; externally peer reviewed.

Data availability statement Data are stored on our internal, high-security server. A deidentified data set is available by applying to the corresponding author.

Supplemental material This content has been supplied by the author(s). It has not been vetted by BMJ Publishing Group Limited (BMJ) and may not have been peer-reviewed. Any opinions or recommendations discussed are solely those of the author(s) and are not endorsed by BMJ. BMJ disclaims all liability and responsibility arising from any reliance placed on the content. Where the content includes any translated material, BMJ does not warrant the accuracy and reliability of the translations (including but not limited to local regulations, clinical guidelines, terminology, drug names and drug dosages), and is not responsible for any error and/or omissions arising from translation and adaptation or otherwise.

Open access This is an open access article distributed in accordance with the Creative Commons Attribution Non Commercial (CC BY-NC 4.0) license, which permits others to distribute, remix, adapt, build upon this work non-commercially, and license their derivative works on different terms, provided the original work is properly cited, appropriate credit is given, any changes made indicated, and the use is non-commercial. See: http://creativecommons.org/licenses/by-nc/4.0/.

\section{ORCID iDs}

Yingxi Zhao http://orcid.org/0000-0002-4937-4703

Gengli Zhao http://orcid.org/0000-0001-8070-9690

\section{REFERENCES}

1 Furman L. Kangaroo mother care 20 years later: connecting infants and families. Pediatrics 2017;139:e20163332.

2 World Health Organization. WHO recommendations on interventions to improve preterm birth outcomes [Internet], 2015. Available: https:// www.who.int/reproductivehealth/publications/maternal_perinatal_ health/preterm-birth-guideline/en/ [Accessed 04 Dec 2019].

3 Chawanpaiboon S, Vogel JP, Moller A-B, et al. Global, regional, and national estimates of levels of preterm birth in 2014: a systematic review and modelling analysis. Lancet Glob Health 2019;7:e37-46.

4 Zhang $Y$, Deng Q, Zhu B, et al. Neonatal intensive care nurses' knowledge and beliefs regarding kangaroo care in China: a national survey. BMJ Open 2018;8:e021740.

5 Yue J, Liu J, Williams S, et al. Barriers and facilitators of kangaroo mother care adoption in five Chinese hospitals: a qualitative study. BMC Public Health 2020;20:1234

6 Zhang B, Duan Z, Zhao Y, et al. Intermittent kangaroo mother care and the practice of breastfeeding late preterm infants: results from four hospitals in different provinces of China. Int Breastfeed $J$ 2020;15:64.

7 Thomas DR. A general inductive approach for analyzing qualitative evaluation data. Am J Eval 2006;27:237-46.

8 Engle WA, Tomashek KM, Wallman C, et al. "Late-preterm" infants: a population at risk. Pediatrics 2007;120:1390-401.

9 Pike M, Kritzinger A, Krüger E. Breastfeeding characteristics of late-preterm infants in a kangaroo mother care unit. Breastfeed Med 2017;12:637-44.

10 Hake-Brooks SJ, Anderson GC. Kangaroo care and breastfeeding of mother-preterm infant dyads 0-18 months: a randomized, controlled trial. Neonatal Netw 2008;27:151-9.

11 Giannì ML, Bezze E, Sannino $\mathrm{P}$, et al. Facilitators and barriers of breastfeeding late preterm infants according to mothers' experiences. BMC Pediatr 2016;16:179.

12 Ludington-Hoe SM, Anderson GC, Simpson S, et al. Birth-related fatigue in 34-36-week preterm neonates: rapid recovery with very early kangaroo (skin-to-skin) care. J Obstet Gynecol Neonatal Nurs 1999;28:94-103.

13 Nyqvist KH, Anderson GC, Bergman N, et al. Towards universal kangaroo mother care: recommendations and report from the first European conference and seventh International workshop on kangaroo mother care. Acta Paediatr 2010;99:820-6.

14 Gregson S, Blacker J. Kangaroo care in pre-term or low birth weight babies in a postnatal ward. Br J Midwifery 2011;19:568-77.

15 Charpak N, Angel MI, Banker D. Strategies discussed at the XIIth international on kangaroo mother care for implementation on a countrywide scale. Acta Paediatrica [Internet] 2020.

16 World Health Organization. Kangaroo mother care: a practical guide [Internet], 2003. Available: http://www.who.int/maternal_child_ adolescent/documents/9241590351/en/ [Accessed 21 Oct 2020].

17 Anderzén-Carlsson A, Lamy ZC, Eriksson M. Parental experiences of providing skin-to-skin care to their newborn infant--part 1: a qualitative systematic review. Int J Qual Stud Health Well-being 2014;9:24906.

18 Baley J, COMMITTEE ON FETUS AND NEWBORN. Skin-to-Skin care for term and preterm infants in the neonatal ICU. Pediatrics 2015;136:596-9.

19 Kostandy RR, Ludington-Hoe SM, Cong X, et al. Kangaroo care (skin contact) reduces crying response to pain in preterm neonates: pilot results. Pain Manag Nurs 2008;9:55-65.

20 Muddu GK, Boju SL, Chodavarapu R. Knowledge and awareness about benefits of kangaroo mother care. Indian $J$ Pediatr 2013;80:799-803. 